\title{
El ejercicio físico para la salud del hombre
}

\author{
Physical exercise for the health of man
}

Efraín Velasteguí López. ${ }^{1}$ Lic. Joel García Espinosa. ${ }^{2}$ Est. Adieren Suarez Cruz. ${ }^{3}$ Est. Yissisleidys Zamora Alonso. ${ }^{4}$ Est. Elienay Romero Sánchez. ${ }^{5}$ \& Est. Karen Sosa Suárez. ${ }^{6}$

Recibido: 16-04-2019 / Revisado: 18-05-2019 / Aceptado: 20-06-2019 / Publicado: 04-07-2019

DOI: https://doi.org/10.33262/anatomiadigital.v2i3.1123

\begin{abstract}
.
The physical activity has been certainly a way of the man's prehistoric behavior. That has always had the movement necessity. Physical exercise is the group of actions muscular and skeletal motorboats. Commonly he/she refers to any physical activity that improves and it maintains the physical aptitude, the health and the person's well-being. For that reason the objective of this work is not more than to argue the
\end{abstract}

\section{Resumen.}

La actividad física ha sido ciertamente un modo de comportamiento prehistórico del hombre., que siempre ha tenido la necesidad de movimiento. Ejercicio físico, es el conjunto de acciones motoras musculares y esqueléticas. Comúnmente se refiere a cualquier actividad física que mejora y mantiene la aptitud física, la salud y el bienestar de la persona. Por eso el objetivo de este trabajo no es más que argumentar la

${ }^{1}$ Ciencia digital, Ambato, Ecuador, luisefrainvelastegui@ cienciadigital.org 7353-0134

2 Ciencias Médicas Mayabeque, Mayabeque, Cuba, jgarcia@infomed.sld.cu iD https://orcid.org/0000-00027353-0135

${ }^{3}$ Ciencias Médicas Mayabeque, Mayabeque, Cuba, asuarez@infomed.sld.cu iD https://orcid.org/0000-00027353-0136

${ }^{4}$ Ciencias Médicas Mayabeque, Mayabeque, Cuba, yzamora@infomed.sld.cu iD https://orcid.org/0000-00027353-0137

${ }^{5}$ Ciencias Médicas Mayabeque, Mayabeque, Cuba, eromero@infomed.sld.cu iD https://orcid.org/0000-00027353-0138

${ }^{6}$ Ciencias Médicas Mayabeque, Mayabeque, Cuba, ksosa@infomed.sld.cu iD https://orcid.org/0000-00027353-0139 
importance of the physical exercise for the man's health by means of the gathering of data in sources of information either digital or in any other format it stops this way to demonstrate its relevance in the human health. They will be given answer to several queries like: What exercise type should I carry out?, Which is the appropriate intensity? among others. The sport has a great influence in the prevention of many illnesses like the obesity, the hypertension and the diabetes. They prolong the person's socially useful time; when improving their physical, cardiovascular, bony and muscular capacity it elevates their productive levels, for what slows the changes characteristic of the age. It assures a bigger work capacity and it assures the longevity when favoring the elimination of toxins and oxidizers. It improves the person's physical aspect.

Keywords: Exercise, health, well-being, man. importancia del ejercicio físico para la salud del hombre mediante la recolección de datos en fuentes de información ya sea digitales o en cualquier otro formato para así demostrar su relevancia en la salud humana. Se darán respuesta a varias interrogantes como: ¿Qué tipo de ejercicio debo realizar?,¿Cuál es la intensidad adecuada? entre otras. El deporte tiene una gran influencia en la prevención de muchas enfermedades como la obesidad, la hipertensión y la diabetes. Prolongan el tiempo socialmente útil de la persona; al mejorar su capacidad física, cardiovascular, ósea y muscular eleva sus niveles productivos, por lo que retarda los cambios propios de la vejez. Asegura una mayor capacidad de trabajo y asegura la longevidad al favorecer la eliminación de toxinas y oxidantes. Mejora el aspecto físico de la persona.

Palabras claves: Ejercicio, salud, bienestar, hombre.

\section{Introducción.}

El hombre, desde la prehistoria, siempre ha tenido la necesidad de movimiento. La actividad física ha sido ciertamente un modo de comportamiento prehistórico del hombre. Es un instinto animal primitivo lo que en principio mueve al hombre hacia el ejercicio físico. Prehistóricamente, los motivos que movían al hombre a practicar el ejercicio físico, midiendo sus fuerzas con los demás, eran la búsqueda de la seguridad, de la subsistencia y de la potencia. La supervivencia del grupo dependía de que sus miembros poseyeran agilidad, fuerza, velocidad y energía. En un principio aparecen unas prácticas corporales relacionadas con la religión y la caza; pero con la aparición de los excedentes de producción comienza el componente lúdico-recreativo-competitivo. Por último, con el surgimiento de los primeros poblados, aparece el componente guerrero Los aztecas medían sus fuerzas luchando y llevando pesos, practicando carreras, lanzamientos y saltos.

En Mesopotamia se ejercía la actividad física como destreza militar. En Japón, la actividad física se fundamentaba en distintas luchas (sumo, judo, kendo) y tiro con arco (kyudo). En 
Grecia el fin supremo de la educación física era crear hombres de acción. Se perseguía el desarrollo físico integral. La educación de los niños entre los 14 y los 18 años era principalmente deportiva, practicando la agonística (ciencia de los combates), carreras de velocidad, esgrima, lanzamientos y pentatlón. Galeno es el primero que propone ejercicios específicos para cada parte del cuerpo: ejercicios para dar tono muscular (cavar, llevar pesos, trepar la cuerda, fuerza y resistencia), ejercicios rápidos (carrera, golpear, juego con pelotas, velocidad y reflejos), ejercicios violentos (repetición de los primeros de forma rápida, potencia). Galeno fue también el primero en utilizar el pulso como medio de detectar los efectos del ejercicio físico. De la época visigoda conocemos algunos ejercicios físicos tales como saltos, esgrima, lanzar jabalina y lucha.

Con el Renacimiento Humanístico se tiende a lo clásico y a lo natural. Se comienza a considerar el ejercicio físico como parte de la educación. El deporte al servicio y el carácter era el lema. Aparece la figura de Rousseau, que defiende una actividad física en el medio natural, en la que el niño se reencuentre con sus orígenes; su pensamiento físico-educativo influirá directamente en gran parte de los profesionales de la educación física de los siglos XIX y XX, muy especialmente en aquellos que defendían los métodos naturales, representados fundamentalmente por la gimnasia natural austriaca, y el método natural de Hebert.

El ejercicio físico es importante para mantener la condición física, puede contribuir positivamente al mantenimiento de un peso saludable, al desarrollo y mantenimiento de la densidad ósea, fuerza muscular y movilidad articular; promueve numerosos procesos. Este es fundamental para el mantenimiento de la salud y es además un poderoso medio de satisfacciones personales. Sin embargo, hoy en día, el automatismo que prevalece en las sociedades industrializadas ha supuesto un terrible ataque al equilibrio cuerpo-espíritu del ser humanos. Las primeras muestras de la falta de ejercitación se evidencian en el sobre peso y en la imposibilidad de realizar tareas antes fáciles y rutinarias. Resulta familiar el caso de la señora que un día descubre que ya no es capaz de subir las escaleras para llegar a su departamento sin tener que hacer una "escala" en el segundo piso y sin sufrir unas palpitaciones que espantan. $\mathrm{O}$ el del señor que ya no puede agacharse para amarrarse las agujetas porque "algunos" kilos de más se lo impiden. En la actualidad, en el mundo existe una tendencia muy marcada en la planificación de las cargas físicas del entrenamiento deportivo el aspecto energético del metabolismo del organismo, esa tendencia está basada en los resultados de investigaciones realizadas en los últimos años especialmente en los EE.UU., Rusia y Alemania. Teniendo en cuenta que el organismo del hombre tiene la posibilidad de regenerar energías que se gastan durante la realización de los ejercicios físicos, incluso incrementadas y es precisamente durante el descanso donde se produce fundamentalmente esta regeneración de energías componente de la carga física, el cual hay que planificarlo y biológicos (ej. metabolismo, sistema inmune) proporcionando un sistema fisiológico saludable. 


\section{Desarrollo}

Se considera ejercicio físico, al conjunto de acciones motoras musculares y esqueléticas. Comúnmente se refiere a cualquier actividad física que mejora y mantiene la aptitud física, la salud y el bienestar de la persona. Se lleva a cabo por diferentes razones como el fortalecimiento muscular, mejora del sistema cardiovascular, desarrollar habilidades atléticas, deporte, pérdida de grasa o mantenimiento, así como actividad recreativa. Las acciones motoras pueden ser agrupadas por la necesidad de desarrollar alguna cualidad física como la fuerza, la velocidad, la resistencia, la coordinación, la elasticidad o la flexibilidad. El ejercicio físico puede estar dirigido a resolver un problema motor concreto. El ejercicio físico mejora la función mental, la autonomía, la memoria, la rapidez, la "imagen corporal" y la sensación de bienestar, se produce una estabilidad en la personalidad caracterizada por el optimismo, la euforia y la flexibilidad mental.

Los ejercicios pueden ser clasificados de la siguiente manera: generales, especiales, competitivos. Los ejercicios generales son aquellos que en su ejecución no contienen fases y/o partes técnicas de la especialidad deportiva seleccionada y están encaminados a la preparación del organismo para las acciones generales del entrenamiento. El desarrollo de las capacidades físicas generales. La recuperación del organismo de las cargas recibidas.

Los ejercicios especiales: estos contienen en su ejecución partes o fases técnicas de la especialidad deportiva seleccionada y están encaminados a la preparación del organismo para las acciones específicas de la parte principal del entrenamiento. Para la enseñanza y corrección de fases y partes de la técnica del deporte seleccionado. Desarrollar las capacidades físicas especiales, teniendo como base la acción motriz del deporte seleccionado.

Los ejercicios competitivos: son aquellos que se ejecutan dentro del juego, los cuales están reglamentados y son los que se desarrollan para obtener los resultados esperados.

Los ejercicios, teniendo en cuenta la dirección del trabajo muscular se pueden clasificar como: flexores y extensores, según su función y objetivos están encaminados al desarrollo de capacidades y habilidades, así como también a la terapéutica de lesiones producidas.

La práctica de ejercicios físicos consume energía y requiere por tanto el aporte de oxígeno y nutrientes a los tejidos. El ejercicio físico practicado de forma regular y frecuente estimula el sistema inmunológico ayudando a prevenir las llamadas enfermedades de la civilización, como la enfermedad coronaria, cardiovascular, diabetes tipo 2 y obesidad. También mejora la salud mental, ayuda a prevenir estados depresivos, desarrolla y mantiene la autoestima, e incluso puede aumentar la libido y mejorar la imagen corporal. El ejercicio físico es importante para mantener la condición física, y puede contribuir positivamente al mantenimiento de un peso saludable, al desarrollo y mantenimiento de la densidad ósea, fuerza muscular y movilidad articular; promueve numerosos procesos biológicos (ej. 
metabolismo, sistema inmune) proporcionando un sistema fisiológico saludable, lo que reduce los riesgos quirúrgicos; reduce los niveles de cortisol, causantes de numerosos problemas de salud tanto físicos como mentales. Tanto el trabajo aeróbico como el anaeróbico aumenta la eficiencia mecánica del corazón mediante el aumento del volumen cardiaco (entrenamiento aeróbico) o el espesor del miocardio (entrenamiento de fuerza). Estos cambios son en general beneficiosos y saludables si se producen como respuesta al ejercicio.

No todos se benefician por igual del ejercicio. Hay una gran variación en la respuesta individual al entrenamiento, condicionada por factores genéticos, donde la mayoría de la población verá un aumento moderado de la resistencia aeróbica, algunos individuos hasta el doble de su consumo de oxígeno, mientras que otros nunca podrán aumentar la resistencia. Sin embargo, el entrenamiento hipertrófico muscular de resistencia está determinado, al menos en gran parte, por la dieta y los niveles de testosterona. Esta variación genética que provoca distintas respuestas en diferentes individuos explica las diferencias fisiológicas entre los atletas de élite y la población general.

Los estudios demuestran que el ejercicio en jóvenes y adultos conduce a una mejor habilidad y condición física y fisiológica en posteriores etapas de la vida. Las alteraciones o enfermedades en las que se ha demostrado que el ejercicio físico es beneficioso, entre otras son: asma, estrés de embarazo, infarto, diabetes mellitus y diabetes gestacional; obesidad, hipertensión arterial, osteoporosis y distintos tipos de cáncer, como el cáncer de próstata y el cáncer colorrectal. Los estudios demuestran una relación fuerte entre la práctica del deporte y el mejoramiento de aspectos emocionales como la autoestima (Escala Rosenberg) y aspectos físicos como reducción de peso, optimización de destrezas motoras y de movilidad articular. Existe una relación muy estrecha entre el cuerpo y la mente.

Beneficios de los ejercicios físicos: La práctica de ejercicio físico aporta a nuestra salud beneficios (fisiológicos, sobre el corazón, sobre el sistema circulatorio, sobre el aparato locomotor, sobre el metabolismo y sobre aspectos psicológicos y psicosociales). Todo ello se analiza en las distintas etapas de la vida: niños y adolescentes, adultos y ancianos.

Beneficios fisiológicos: Mayor capacidad cardiorrespiratoria, que permite optimizar el funcionamiento general del organismo y su rendimiento frente a demandas específicas. Menor riesgo de enfermedades coronarias, así como prevención de segundos episodios de ataques cardiacos en personas que han sufrido infartos. Disminución de la tensión arterial y prevención de la hipertensión arterial. Prevención y disminución de trastornos respiratorios. Prevenir el deterioro de la mineralización de los huesos y la atrofia en el tejido muscular, el acortamiento, así como la rigidez de las articulaciones. Mayor flexibilidad a cualquier edad. Prevenir y controlar el dolor muscular (miositis y mialgias). Reducir peso y mantener el peso ideal: aumento de la masa muscular y descenso de la masa grasa. Mejorar la apariencia física 
en general. Además, ayuda a tomar conciencia de las fuerzas y limitaciones físicas del individuo, facilitando la propiocepción y dando un buen sentido del propio cuerpo.

Sobre el corazón: Disminuye la frecuencia cardiaca en reposo. Durante el esfuerzo aumenta la cantidad de sangre que expulsa el corazón en cada latido, así la eficiencia cardiaca es mayor "gastando" menos energía. Estimula la circulación coronaria dentro del músculo cardiaco favoreciendo la "alimentación" del corazón.

Sobre el sistema circulatorio: Contribuye a la reducción de la tensión arterial. Favorece y aumenta la circulación en todo el organismo. Disminuye la formación de coágulos dentro de los vasos sanguíneos, previniendo la aparición de infartos, trombosis y embolias. Actúa sobre el endotelio vascular, mejorando su actividad y manteniéndolo más elástico. Mejora el retorno venoso previniendo, entre otros cuadros, la aparición de varices.

Sobre el aparato locomotor: Favorece el fortalecimiento de estructuras articulares (huesos, cartílagos, ligamentos, tendones) y mejora la función del sistema músculo esquelético contribuyendo en la calidad de vida y grado de independencia especialmente entre las personas de edad. Aumenta la elasticidad muscular y articular. Incrementa la fuerza y resistencia de los músculos. Previene la aparición de osteoporosis. Previene el deterioro muscular producido por los años. Facilita los movimientos y el desarrollo de las actividades de la vida diaria.

Sobre el metabolismo: Aumenta la capacidad de aprovechamiento del oxígeno que le llega al organismo por la circulación. Aumenta la actividad de las enzimas musculares, permitiendo un mejor metabolismo del músculo con una menor necesidad de exigencia de trabajo cardíaco. Mejora la respuesta inmunológica ante infecciones o agresiones de distinto tipo. Incrementa la secreción y trabajo de diferentes hormonas que contribuyen a la mejoría de las funciones del organismo. Aumenta el consumo de grasas durante la actividad con lo que contribuye a la pérdida de peso. Colabora en la disminución del colesterol total y del colesterol LDL ("malo") con aumento del colesterol HDL ("bueno"). Mejora la tolerancia a la glucosa favoreciendo el tratamiento de la diabetes. Facilita la digestión, favorece el tránsito intestinal regulando hábitos de evacuación y evitando el estreñimiento, disminuyendo el riesgo de cáncer de colon.

Sobre los aspectos psicológicos: Aumenta la sensación de bienestar y disminuye el estrés mental (se produce liberación de endorfinas que favorecen el "sentirse bien" después del ejercicio). Aumento y fortalecimiento de la autoconfianza y la autoestima. Mejora la imagen de uno mismo contribuyendo al establecimiento de una relación positiva con el propio cuerpo. Disminuye el grado de agresividad, ira, ansiedad, angustia y depresión. Disminuye la sensación de fatiga, dando más energía y capacidad de trabajo. Mejora la calidad del sueño y disminuye el insomnio. Colabora en el mantenimiento de una vida sexual plena. 
Sobre los aspectos psico-sociales: Alivia estados de ánimo adversos y otras manifestaciones de estrés psicológico. Aporta divertimento, distracción de problemas diarios...Previene y disminuye síntomas de estados patológicos de ansiedad (trastornos de ansiedad generalizada, trastorno fóbico, trastorno obsesivo compulsivo) y depresión. Contribuye a prevenir y modificar ciertas conductas anómalas, como la conducta antisocial. Interviene en la prevención, control y abandono de comportamientos perjudiciales para la salud como el alcoholismo, hábito tabáquico y drogadicción. Ayuda a desarrollar un estilo de vida basado en el compromiso y perseverancia, el optimismo, la cooperación...Establece valores y modelos positivos. Desarrollo y fortalecimiento de relaciones sociales. Aprendizaje y perfeccionamiento de habilidades deportivas. Afrontamiento y consecución de retos.

La higiene en el ejercicio físico: Son las normas que hay que cumplir antes, durante y después del ejercicio físico para así prevenir posibles consecuencias negativas. Las normas son: Antes del ejercicio, uso de instalaciones, materiales y vestuarios. Durante el ejercicio, sobre la práctica física, características del ejercicio y del individuo. Después del ejercicio, relajarse y aseo personal.

Para conseguir una vida sana es necesario realizar ejercicios corporales, tener hábitos alimenticios y mantener una higiene deportiva y corporal, y de este modo alcanzaremos un buen estado tanto físico como mental. La higiene deportiva significa mantener una serie de hábitos que ayudan a obtener el máximo rendimiento con el ejercicio físico y a realizarlo con la máxima garantía, y dentro de ella es imprescindible la higiene corporal.

Dentro de esta serie de hábitos podemos distinguir tres etapas a tener en cuenta: Condiciones previas al ejercicio: el correcto punto de partida para llevar a cabo cualquier ejercicio físico es una revisión médica, que nos ayudará a detectar cualquier posible anomalía de nuestro organismo. Nunca haremos coincidir la práctica del ejercicio con la digestión de la comida y siempre se realizará un calentamiento previo, suave y progresivo. Condiciones durante el ejercicio: utilizaremos material apropiado a la actividad y la práctica del ejercicio se hará con la intensidad adecuada al nivel de condición física, bebiendo líquido durante todo el esfuerzo. Condiciones posteriores al ejercicio: el ejercicio se acabará con unos minutos de actividad física suave para acelerar la recuperación, una hidratación correcta (bebiendo pequeñas, pero frecuentes cantidades) y la higiene corporal correspondiente. Esta higiene corporal incluye un conjunto de prácticas destinadas a mantener una correcta limpieza del cuerpo. La falta de higiene trae como consecuencia la aparición de enfermedades en todos los sistemas, por eso, es importantísimo seguir las normas recomendadas: Bañarse o ducharse diariamente, antes (si va a usar una piscina) y después de realizar una actividad física.

Secarse los pies correctamente después de cada lavado y aplicar polvos de talco si ha habido una sudoración excesiva. Se recomienda el uso de medias $100 \%$ de algodón. No usar prendas como anillos, relojes, manillas, pulsos, argollas que puedan ocasionar un accidente. Los implementos deportivos siempre deberán ser completamente personales e intransferibles, 
para evitar transmisión de enfermedades entre los deportistas. Usar ropa holgada y de algodón. Usar calzado cómodo y adecuado. No excederte los primeros días. Ingerir agua durante la actividad física según la temperatura del local o necesidades del organismo. No embucharse. Seleccione el ejercicio físico de acuerdo a sus características incluyendo enfermedades si la padece. Tener la mente acondicionada para la actividad física. Realizar ejercicio aeróbico de baja intensidad y larga duración.

Entrenar por la mañana es más recomendable desde el punto de vista del consumo calórico porque produce un efecto acelerador del metabolismo durante las horas posteriores.

Pequeños consejos para lograr que la actividad física forme parte de su vida: camine en lugar de usar medios de transporte: si utiliza un transporte público, bájese un par de paradas antes de su destino. Use menos su automóvil y, cuando lo haga, trate de estacionar en un lugar más alejado que el habitual. Utilice escaleras en lugar del ascensor. Tómese dos minutos por hora en el trabajo o el hogar para "estirar las piernas" y movilizar los músculos. Aproveche los momentos de ocio para movilizar las articulaciones con ejercicios de poca intensidad. Realice un breve paseo a pie antes del desayuno o la cena (o ambos). Cambie 10 minutos de televisión por un par de vueltas a la manzana. Vaya caminando o en bicicleta a realizar las compras simples de todos los días. Saque a pasear a su perro todos los días. Cuando camine, trate de ir acelerando su paso progresivamente. Si puede, elija caminos con subidas y bajadas. Cuando mire televisión, trate de hacerlo sentado en lugar de acostado o tendido en un sofá. Tenga lejos el control remoto para movilizarse cuando quiera cambiar de canal. Hable por teléfono en posición de pie, con mínimos movimientos, en lugar de hacerlo sentado. Haga las cosas por Ud. mismo. No pida que le traigan algo, búsquelas. Si su trabajo le permite una pausa (hora de almuerzo, por ejemplo) utilice 10 minutos para dar un paseo a pie.

¿Que tipo de ejercicio debo realizar? Las caminatas son una buena alternativa para muchas personas, sobre todo después de tiempos prolongados de inactividad. Los ejercicios dinámicos están también incluidos en la natación, el ciclismo, el trote, el golf, el tenis, el remo, el baile. Aquellas personas con capacidad y mejor aptitud física podrán iniciarse con niveles de mayor intensidad e incluir deportes de más exigencia (fútbol, basket, voleibol, etc.).

¿Cuál es la intensidad adecuada? Ya vimos que para alcanzar beneficios para la salud no son necesarios ejercicios vigorosos. Existen diferentes formas para medir la intensidad del esfuerzo. Quizás la más frecuentemente utilizada sea el registro de los latidos cardíacos por minuto (frecuencia cardíaca). Los ejercicios de moderada intensidad se desarrollan con niveles de frecuencia cardíaca entre el 50 a $75 \%$ de la máxima que corresponde a la persona.

¿Cómo tomar el pulso? Los accesos más fáciles son: El cuello (pulso carotídeo). Deben apoyarse ( $\sin$ apretar) los dedos mayor e índice sobre la zona lateral del cuello, $2 \mathrm{~cm}$, aproximadamente, por debajo de la mandíbula. El canal del pulso en la zona donde se 
flexiona la muñeca del lado del pulgar. Los mismos dedos se apoyan sobre una hendidura (canal) donde es posible palpar el latido de la arteria radial. Una vez localizado el pulso deben contarse los latidos durante 6 segundos y multiplicar por 10. Ese es el valor de la frecuencia cardíaca por minuto. Es importante que al realizar las actividades se sienta placer y no sufrimiento. Esto significa que si aparecen síntomas como fatiga o falta de aire se entienda que el ejercicio está siendo demasiado fuerte y tal vez sea necesario disminuir su intensidad.

¿Cuánto tiempo y con qué frecuencia se deben realizar los ejercicios? Se debe tener como meta realizar actividad física de moderada intensidad durante 30 a 40 minutos todos los días. Sin embargo, para muchas personas esta frecuencia puede ser muy difícil de planificar. Es posible, entonces, programar un ejercicio progresivo con tiempos e intensidades que se irán incrementando. Una de las formas de iniciar el programa consiste en la realización durante 3 veces por semana de planes de caminatas. La iniciación es con un tiempo total de 30 minutos divididos de la siguiente forma: 1) 8 minutos de entrada en calor (incluye caminata lenta inicial de 3 minutos; 2) 15 minutos de caminatas a ritmo moderado a nivel 3 de la escala o $60 \%$ de la máxima frecuencia cardíaca teórica; 3) vuelta a la calma con ejercicios de elongación. El tiempo de ejercicio se incrementa cada semana para llegar a los 60 minutos totales con 10 minutos de entrada en calor, 40 minutos de caminatas y 10 de vuelta a la calma y elongación.

Estructura de una sección de ejercicios: Un programa estructurado de actividad física puede dividirse en las siguientes tres fases: Entrada en calor el ejercicio propiamente dicho (caminata, trote, bicicleta. baile o el deporte elegido, ejercicios de fuerza muscular, recreación)

Vuelta a la calma (elongación) Ya hemos visto las características de la segunda fase. A continuación se describirán algunos de los ejercicios más utilizados para flexibilidad y elongación que forman parte, junto con la caminata inicial, de la importante fase de "entrada en calor". En la "vuelta a la calma", los movimientos pueden ser similares. Esta última fase tiene también gran importancia en la prevención de lesiones, adaptación cardiovascular y regeneración de las sustancias que aportan la energía al músculo que trabaja.

Entrada en calor todas las actividades dinámicas deben estar precedidas por movimientos de calentamiento y elongación de articulaciones y grupos musculares. Tienen una importancia fundamental para prevenir lesiones y preparar al aparato cardiovascular para el ejercicio. Cada ejercicio tendrá una duración de 10 a 12 segundos y se repetirá en el sentido contrario o con el miembro superior o inferior del lado opuesto. Los movimientos deben realizarse lentamente y sin" rebotes" o insistencias. En personas de mayor edad o muy desacondicionadas, los movimientos no deben ser extremos, sino simplemente llegar hasta donde se puede, sin sufrir dolores. Los números de series y las repeticiones son aproximadas y deben adaptarse a las condiciones de cada persona. Estos ejercicios pueden utilizarse también como actividad única en varios momentos del día. Se describen varios tipos de 
ejercicios. Podrán elegirse unos u otros tratando de involucrar a la mayor parte de los grupos musculares.

El mejor ejercicio: Elija una actividad que le permita lograr el desarrollo de una musculatura equilibrada y mejorar el funcionamiento cardiovascular y el metabolismo, pero evite aquella actividad que dé lugar a nuevas patologías, por una ejercitación exagerada o defectuosa.

Los ejercicios de tipo aeróbico son una buena opción. Estos ejercicios se caracterizan por implicar un movimiento continuo y permanente durante un mínimo de 20 minutos. Es el caso del trote o de la caminata. Para que estos ejercicios resulten saludables deben realizarse a una frecuencia cardiaca del 50\% de la capacidad del individuo, y deben acompañarse de una respiración acompasada. Un indicador de que no se está forzando demasiado al organismo, es que la persona que se ejercita puede entablar una conversación si así lo desea.

\section{Conclusiones.}

- Los ejercicios físicos provocan efectos en el organismo y están encaminados al desarrollo de capacidades y habilidades. Además, el deporte tiene una gran influencia en la prevención de muchas enfermedades como la obesidad, la hipertensión y la diabetes. Prolongan el tiempo socialmente útil de la persona; al mejorar su capacidad física, cardiovascular, ósea y muscular eleva sus niveles productivos, por lo que retarda los cambios propios de la vejez. Asegura una mayor capacidad de trabajo y asegura la longevidad al favorecer la eliminación de toxinas y oxidantes. Mejora el aspecto físico de la persona. Mejora el sistema inmune. Regula todos los índices relacionados con hipertensión, glucemia y grasas en la sangre. Mejora la calidad del sueño. Mejora y fortalece el sistema osteomuscular (tendones) contribuyendo al aumento de la calidad de vida y grado de independencia especialmente entre las personas con más edad.

\section{Referencias bibliográficas.}

Conill, D. (2012). Los principios del ejercicio físico.Globedia http://cu.globedia.com/principios-ejercicio-fisico\#

Cintra Cala, O. y Balboa Navarro, Y. (2011). La actividad física: un aporte para la salud. Lecturas Educación Física y Deportes, 159, 1. http://www.efdeportes.com/efd159/laactividad-fisica-para-la-salud.htm

Durán Montero, F. y Leyva Paján, C. (2014). Generalidades e importancia del ejercicio físico para la salud. Lecturas Educación Física y Deportes, 189, 1.http://www.efdeportes.com/efd189/importancia-del-ejercicio-fisico-para-lasalud.htm 
Balboa Navarro, Y.; Cintra Cala, O y BoschenBaldrich, A. (2011). La obesidad: ¿salud u obsesión? Lecturas Educación Física y Deportes, 152, 1.http://www.efdeportes.com/efd152/la-obesidad-salud-u-obsesion.htm

Calderón, C. y colectivo. Fundamentos generales de la teoría y metodología de la Educación Física. Ed. Pueblo y Educación. La Habana. 1993.

Cintra Cala, O. (2014). Influencia de un programa de actividades físicas en el mejoramiento de la salud de los trabajadores de la Facultad "Dr. Salvador Allende",Revista Cubana de Tecnología de la Salud 


\section{PARA CITAR EL ARTÍCULO INDEXADO.}

Velasteguí López, E., García Espinosa, J., Suarez Cruz, A., Zamora Alonso, Y., Romero Sánchez, E., \& Sosa Suárez, K. (2019). El ejercicio físico para la salud del hombre. Anatomía Digital, 2(3), 86-97. https://doi.org/10.33262/anatomiadigital.v2i3.1123

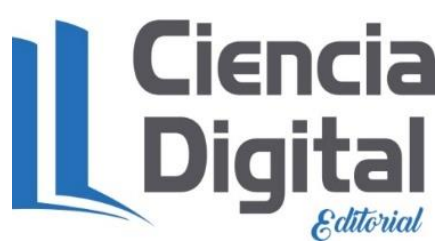

El artículo que se publica es de exclusiva responsabilidad de los autores y no necesariamente reflejan el pensamiento de la Revista Anatomía Digital.

El artículo queda en propiedad de la revista y, por tanto, su publicación parcial y/o total en otro medio tiene que ser autorizado por el director de la Revista Anatomía Digital.
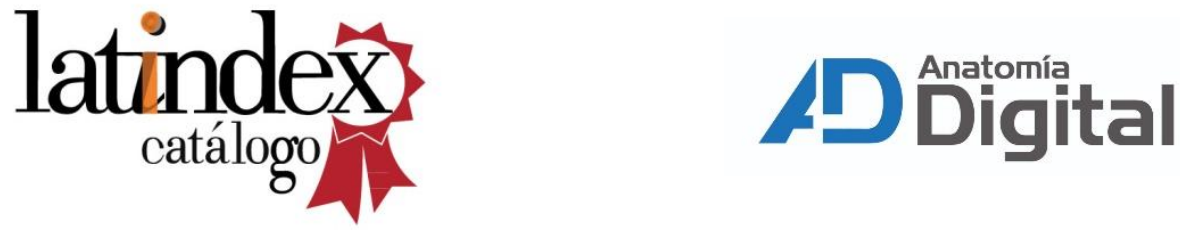